\title{
IMPACTOS DEL TURISMO RESIDENCIAL PERCIBIDOS POR LA POBLACIÓN LOCAL: UNA APROXIMACIÓN CUALITATIVA DESDE LA TEORÍA DEL INTERCAMBIO SOCIAL
}

\author{
Local Perceptions of the Impacts of Second-Home Tourism: A Qualitative Approach based on Social- \\ Exchange Theory
}

\author{
Carlos Monterrubio \\ Ana Pricila Sosa-Ferreira \\ Maribel Osorio-García
}

Resumen: Desde la teoría del intercambio social, se analizan los impactos del turismo residencial en Puerto Morelos, México. Con base en entrevistas a residentes permanentes, se evidencia que el turismo residencial se asocia con el empleo, el efecto multiplicador, la difusión de la cultura, el cuidado del entorno natural y acciones altruistas con locales. El incremento del precio de los inmuebles y de la competencia empresarial son considerados costos del fenómeno. Los pobladores realizan una valoración de los beneficios económicos y de los costos socioculturales y medioambientales a nivel individual y colectivo para definir su posición frente al turismo residencial.

Palabras clave: impactos del turismo, turismo residencial, teoría del intercambio social.

Abstract: Framed by social-exchange theory, this study aimed to identify the impacts of second-home tourism in Puerto Morelos, Mexico. Based on in-depth interviews with permanent residents, we found that second-home tourism at the destination is associated with employment opportunities, the multiplier effect, cultural promotion, preservation of the natural environment, and altruistic initiatives. The increase in real-estate values and entrepreneurial competition are considered to be costs of the phenomenon. We conclude that, in order to define their stance on second-home tourism, locals consider both individual and community costs and benefits in their analysis of the impacts of this development.

Keywords: tourism impacts, second-home tourism, social-exchange theory.

Carlos Monterrubio. Doctor por the Manchester Metropolitan University, Reino Unido. Profesor-investigador en la Universidad Autónoma del Estado de México. Temas de especialización: aspectos socioculturales del turismo, turismo y sexualidad y turismo como campo de estudio. Correo electrónico: jcmonterrubio@yahoo.com.mx. Ana Pricila Sosa Ferreira. Doctora en Geografía por la Universidad Nacional Autónoma de México. Profesora-investigadora en la Universidad del Caribe, Cancún, México. Temas de especialización: turismo, economía y sociedad, turismo en el Caribe mexicano. Correo electrónico: psosa@ucaribe.edu.mx.
Maribel Osorio García. Doctora en Ciencias Sociales y Políticas por la Universidad Iberoamericana, México. Profesora-investigadora en la Universidad Autónoma del Estado de México. Temas de especialización: estudios socioterritoriales del turismo, sistema y planificación turística y estado del conocimiento del turismo. Correo electrónico: maribelosorio2@gmail.com.

Enviado a dictamen: 24 de abril de 2017. Aprobación: 17 de octubre de 2017. Revisiones: 1 . 
E n las últimas décadas el tema de los impactos ha dominado en los estudios turísticos. Empíricamente se ha evidenciado que el turismo tiene repercusiones relevantes en los entornos económico, sociocultural y natural, pero también se ha demostrado que éstas dependen considerablemente del tipo específico de actividad turística. Como una modalidad particular, el turismo residencial se ha caracterizado por tener efectos directos en la apropiación del suelo, en la construcción y compraventa de inmuebles, en interacciones interpersonales cercanas, en las relaciones laborales a largo plazo o en la composición demográfica en los destinos, entre otros temas. Pese a su notable presencia en diversas partes del mundo, los impactos de este fenómeno han sido pocas veces objeto de estudio en los denominados países en vías de desarrollo.

En cuanto a los pobladores locales, muy poco se sabe sobre por qué perciben de ciertas formas los cambios que la actividad turística produce y por qué sostienen determinadas actitudes ante el turismo, aunque conocer dichas percepciones puede ser de utilidad en la toma de decisiones o en la generación de recomendaciones para los actores involucrados. Para comprender no sólo el qué, sino el porqué de las percepciones de los impactos, la adopción de ciertas teorías, entre ellas la teoría del intercambio social, es de gran utilidad.

Con la intención de contribuir al estudio y entendimiento del comportamiento social de una comunidad receptora de turismo residencial, se llevó a cabo una investigación en la comunidad de Puerto Morelos, México, para ilustrar la percepción de los impactos de esta actividad en un destino de costa. Específicamente, este artículo tiene como objetivo analizar cómo se perciben los cambios económicos, socioculturales y en el entorno natural asociados al turismo residencial en la comunidad seleccionada, según la perspectiva de los residentes permanentes y a partir de la teoría del intercambio social. Se aplicaron técnicas cualitativas de investigación que permiten profundizar en la información recibida de los residentes sobre su percepción en relación con el turismo, y procesarla.

\section{Marco teórico-conceptual}

\section{Teoría del intercambio social}

Aunque su definición ha sido cuestionada (Emerson, 1976), la teoría del intercambio social es considerada como una de las más influyentes en la explicación del comportamiento social desde la sociología y la psicología social. Si bien existen distintas perspectivas sobre esta teoría, se ha coincidido ampliamente en que el intercambio social comprende acciones interdependientes supeditadas a las reacciones satisfactorias de otros. De acuerdo con Cropanzano y Mitchell (2005), los intercambios entre los individuos se guían por reglas y normas establecidas de manera tácita o explícita que suelen ser, principalmente, de reciprocidad o negociación. En su mayoría, las reglas de reciprocidad suelen dominar; es decir, los intercambios sociales se generan por la esperanza de recibir algo a cambio de cierto comportamiento. Aunque la aplicación y las expectativas del intercambio varían de una cultura a otra, la norma de reciprocidad puede ser considerada un principio universalmente esperado en cualquier interacción social. Por su parte, las reglas negociadas suelen ser más explícitas y frecuentemente son parte de transacciones económicas. Pero los intercambios sociales no se dan únicamente a partir de la reciprocidad y la negociación, pues existen otras razones por las cuales los seres humanos intercambian socialmente, como la racionalidad, el altruismo y la competencia.

Aunque el intercambio ha sido analizado desde los recursos, particularmente desde el valor económico de éstos, el intercambio social también se origina a partir de valores simbólicos, es decir, no está condicionado únicamente por bienes materiales, por lo que el valor de los recursos no siempre es el mismo.

La teoría del intercambio social ha sido una de las más aceptadas y adoptadas en la explicación de las actitudes de los residentes locales hacia el turismo (Jurowski y Gursoy, 2004; Haley, Snaith y Miller, 2005), aunque, desde el conocimiento de los autores de este artículo, no se ha utilizado en el contexto del turismo residencial. En el marco de la actividad turística, la teoría 
postula que los residentes que perciben el turismo como una actividad valiosa para sus vidas y que sus costos no exceden los beneficios que produce, tienen una actitud favorable hacia la actividad y apoyan su desarrollo. En este sentido, diversos autores han postulado que, a nivel individual, los residentes empleados y que reciben más beneficios de la industria turística tienen actitudes más positivas hacia los turistas y hacia el turismo en sí.

Para analizar los impactos del turismo y las actitudes locales hacia la actividad es importante distinguir entre los turistas y el turismo como tal. Las actitudes hacia los turistas derivan principalmente de las interacciones y los intercambios sociales entre ellos y los locales, y son de carácter más personal; por otra parte, las actitudes hacia el turismo suelen originarse a partir de los cambios percibidos en el entorno económico, sociocultural y físico como consecuencia del fenómeno, y se construyen en función de la valoración del beneficio o del costo comunitario. De esta forma, en un mismo grupo social las actitudes hacia los turistas no son necesariamente las mismas que hacia el turismo como fenómeno multidimensional. Así, según Jurowski y Gursoy (2004), la aceptación de los cambios que el fenómeno genere en las poblaciones locales dependerá en parte de los beneficios que los residentes reciban en comparación con los costos. La teoría ha servido de base para muchos estudios de caso tanto en América como en Europa, ${ }^{1}$ y su aportación ha sido útil a pesar de las críticas que ha recibido (Sharpley, 2015). En publicaciones recientes se ha postulado que el contexto socioeconómico en el que se busque analizar los beneficios y costos del turismo influirá considerablemente en las percepciones locales. De acuerdo con Ward y Berno: "puede esperarse que los residentes de países en vías de desarrollo perciban mayores beneficios económicos del turismo y, por tanto, tengan actitudes más favorables hacia el [mismo]" (2011: 1558). La comprobación empírica de esta postulación, no obstante, se encuentra aún pendiente.

\section{Impactos del turismo}

El término "impacto" ha sido uno de los más utilizados en el estudio del turismo y del cambio en las estructuras locales. En estos ámbitos, se consideran impactos los cambios tangibles e intangibles suscitados en las meso y macroestructuras económicas, sociales y naturales como consecuencia del turismo en una localidad, y tienden a ser clasificados en positivos y negativos. Así, comúnmente se han considerado como impactos positivos la generación de empleo, la revitalización de artesanías y tradiciones o la conservación del patrimonio, entre muchos otros; y se han reportado como impactos negativos la sobredependencia de la actividad, la saturación en espacios públicos, el incremento del tránsito vehicular o la modificación de la identidad cultural, por mencionar algunos (Mason, 2008)

Desde la teoría del intercambio social, la valoración que los locales hagan sobre estos impactos determinará su actitud hacia el turismo, aunque es importante tener presente que la calificación de un impacto como positivo o negativo es muchas veces subjetiva. Esto se debe a que dentro de una misma comunidad las personas evalúan los cambios de distintas maneras y, por ello, puede existir desacuerdo en lo que resulta ser deseable o indeseable, especialmente cuando se consideran las condiciones individuales al interior de una localidad. Esta posible variación explica en parte las distintas actitudes hacia el fenómeno. Asimismo, reconocer esta multiplicidad de actitudes exige una reflexión en cuanto a clasificar los impactos como positivos o negativos, como benéficos o perjudiciales, particularmente entre quienes pueden tener posibilidades de intervención, como los investigadores, planificadores, empresarios y otros agentes de acción.

Independientemente del beneficio o perjuicio que la actividad turística genere, los impactos pueden tener un efecto directo o indirecto, tanto a nivel individual como colectivo, sobre los residentes del destino en cuestión. Son varios los factores que se conjugan para generar cambios en cualquiera de los niveles. Uno de ellos, y ciertamente no el único ni siempre el más importante, es el tipo de turista - y con ello el tipo de actividad turística- que se presenta en el destino. Particularmente, el tipo de servicios y productos que se demanden, así como el encuentro y las relaciones que se generan entre el residente y el visitante, suelen definir 
el tipo de cambios. Debe señalarse que no existe un conjunto lineal de relaciones que determine el grado de cambio que se pueda crear; cualquier cambio resultante es un reflejo no sólo de las características económicas y socioculturales de los visitantes, sino también de las condiciones locales (Ryan, 2003).

\section{Turismo residencial}

El turismo residencial constituye una de las modalidades más singulares de la actividad turística ya que implica la estancia temporal en una segunda vivienda, donde se realizan actividades recreativas y de descanso en un destino distinto al de la vida cotidiana. La característica de alojarse en una vivienda, en lugar de en el convencional hotel, imprime un patrón de comportamiento diferente a otras formas de turismo, ya que involucra aspectos de apropiación del suelo, de relaciones laborales directas y de interacciones frecuentes y a largo plazo con la población local, entre otras características, que repercuten de manera particular en aspectos inmobiliarios, migratorios y socioculturales, como los más destacados. El término de turismo residencial fue introducido por Jurdao (1979) a finales de los años setenta en su libro España en venta: compra de suelo por extranjeros y colonización de campesinos en la Costa del Sol, al analizar el cambio de uso y propiedad del suelo en Málaga, fenómeno que ya había sido señalado por Mario Gaviria (1974a y 1974b) cuando inversores extranjeros adquirieron terrenos en destinos turísticos con propósitos de urbanización. Aunque este fenómeno ha sido objeto de estudio desde la década de los setenta, es en el presente siglo cuando su continuo incremento ha generado mayor interés (Huete y Mantecón, 2010), constituyéndose en uno de los temas ascendentes de la investigación turística. Varios estudiosos ${ }^{2}$ han documentado la presencia del turismo residencial en muy distintos países del mundo, dando cuenta de su expansión tanto en países desarrollados, como en desarrollo.

\section{Impactos del turismo residencial}

Los estudios sobre los impactos del turismo residencial han sido ampliamente consistentes y han revelado que, como cualquier otra modalidad, ésta es generadora de efectos diversos en las comunidades locales, especialmente en las costeras. Como una modalidad muy específica, los cambios generados por o asociados a la actividad son también muy específicos, en especial las transformaciones de la dinámica poblacional e inmobiliaria (Hidalgo y Zunino, 2011). En el caso de las costas de España, país en donde los cambios asociados se han mostrado con mayor intensidad (Huete, 2010), la actividad turística residencial ha sido un agente acelerador primordial en el proceso de cambio social.

En España, debido a los procesos migratorios que caracterizan al país, este fenómeno ha causado cambios significativos en la composición demográfica de las localidades y transformaciones importantes en los paisajes. Asimismo, ha guiado hacia la desaparición de actividades tradicionales, hacia la sustitución de la cultura local por una nueva cultura cosmopolita y global, y hacia la constitución de comunidades fragmentadas y nuevas formas de interacción social (Aledo, 2008; Aledo, García y Ortiz, 2010). Los sectores de la construcción, de bienes raíces e inmobiliario son los que más se han visto beneficiados; sin embargo, el consumo del suelo crea problemas por la colisión con otros usos productivos del suelo (Serrano, 2003).

Aunque también se han señalado impactos negativos de este tipo de turismo, algunos estudiosos sugieren que, en términos generales, los beneficios percibidos compensan los costos sociales y medioambientales. Un análisis más detallado sugiere que las percepciones y actitudes de los residentes hacia el fenómeno y los cambios asociados a él permiten identificar grupos específicos a partir de sus cuestionamientos sobre los impactos (Huete, 2010; Mantecón y Huete, 2011). Desde la teoría del intercambio social, se espera entonces que los beneficios económicos del turismo residencial adquieran mayor importancia frente a los costos de éste y que, por tanto, las actitudes locales sean positivas.

En el caso específico de México, aunque ya se ha abordado el tema, existen necesidades de investigación pendientes de atender. En el país han sido pocos los estudios realizados sobre impactos del turismo residencial en particular, aunque, de acuerdo con las 
investigaciones iniciales (Talavera, 1982; CESTURSECTUR, 2004; Hiernaux, 2005 y 2010), éste se manifiesta básicamente en sitios costeros y en las periferias de las grandes áreas metropolitanas. Entre las investigaciones realizadas en sitios costeros destacan los estudios sobre el Centro Integralmente Planeado de Los Cabos (Lizárraga, 2010) y sobre el puerto de Mazatlán (Lizárraga, 2014) y, en cuanto a los destinos rurales de las periferias de los centros urbanos, los de Valle de Bravo (Deverdum, Osorio e Iracheta, 2016) y Malinalco (Ramírez y Osorio, 2010; Escobedo et al., 2015). Aunque algunos otros estudiosos han tratado el tema de manera tangencial (Schafran y Monkkonen, 2011; Negrete, 2011), claramente existe una ausencia de investigaciones que develen los cambios socioculturales generados por el turismo residencial en los centros de playa en México.

\section{Puerto Morelos}

\section{Antecedentes}

Puerto Morelos es un pueblo costero ubicado a treinta kilómetros al sur de Cancún, en el estado de Quintana Roo, México (ver mapa 1); es uno de los puertos más antiguos del Caribe mexicano y el principal puerto industrial de Quintana Roo en la actualidad, aunque en los años sesenta, cuando se analizaba la creación de Cancún, era apenas una aldea en la que habitaban unos cuantos pescadores (Cruz et al., 2013a).

Durante los años sesenta la pesca activó la economía y el repoblamiento de Puerto Morelos en conjunto con otros factores como: la actividad que se generó en los cincuenta y sesenta en Cozumel y Tulum; la mayor comunicación vial tras la construcción de la carretera Cancún-Playa del Carmen-Tulum, y la exportación de maderas tropicales. Estos factores impulsaron la reactivación del puerto y el crecimiento del poblado, que empezó a brindar servicios a los usuarios del puerto en sus largas esperas para cruzar a Cozumel. Muy pronto se empezaron a recibir turistas de Canadá en coordinación con la agencia Folie-tourisme, que promovió Puerto Morelos para visitantes interesados en la naturaleza y la vida saludable. También el pueblo se convirtió en centro de reunión de los académicos que se fueron integrando a los centros de investigación que se instalaron allí a partir de 1964. Estos elementos fueron formando el carácter y la imagen que conserva hasta hoy Puerto Morelos.

A partir del crecimiento de Cancún, tanto Puerto Morelos como Playa del Carmen, en el corredor CancúnTulum, ofrecieron actividades complementarias, principalmente náuticas. Puerto Morelos fue reforzando así su identidad de pequeño poblado, que atrajo a turistas residenciales e inmigrantes nacionales e internacionales que buscaban la tranquilidad y el encanto del puerto, así como a visitantes identificados con la naturaleza y la imagen de "pueblo de pescadores". Pequeños hoteles al principio, y otros medianos y grandes desde finales de los noventa, se fueron instalando en el puerto, junto con pequeños negocios de venta de artesanías y restaurantes.

Actualmente Puerto Morelos tiene una oferta de habitaciones de hotel relativamente pequeña para el contexto del norte de Quintana Roo; su crecimiento ha sido constante, pero sensiblemente menor al de la Riviera Maya, la cual cuenta con 35000 habitaciones. Se caracteriza por tener una oferta diversificada, desde grandes resorts hasta habitaciones disponibles en casas y departamentos, con pequeños hoteles y hoteles boutique, aunque conserva una imagen de baja densidad poblacional y, como se ha mencionado, de "pueblo de pescadores". Su mercado está integrado principalmente por personas que buscan tranquilidad, quienes encuentran en el sitio una amplia variedad de hospedaje para elegir. La exclusividad se relaciona con la baja densidad, el entorno natural y el ambiente de pueblo, con pocas instalaciones lujosas.

Uno de sus principales problemas es la tendencia al crecimiento y la presión de inversionistas que fomentan el crecimiento bajo el modelo masivo, en contraposición a una parte de la comunidad y a empresas que pugnan por la conservación del entorno y por un turismo de baja densidad. De acuerdo con algunas opiniones, el hecho de que Puerto Morelos fuera parte del municipio Benito Juárez, al que pertenece Cancún, impidió que se diera 
mayor impulso al turismo - puesto que los recursos y esfuerzos principalmente se dirigían a Cancún-, lo que tuvo un efecto positivo porque su crecimiento fue menor y más gradual. Sin embargo, recibió poca atención del gobierno municipal y un desarrollo insuficiente en infraestructura, lo que impidió que se aprovechara su potencial para ser, como algunos residentes indican, "otro San Miguel de Allende". Esta situación puede cambiar porque en enero de 2016 la localidad de Puerto Morelos pasó a ser cabecera del municipio del mismo nombre y cuenta ya con su propio plan de desarrollo urbano.

La oferta turística incluye pequeños cafés y restaurantes en la zona centro, y se ofrecen paseos en lancha para buceo y snorkel aprovechando que se ubica, junto con Akumal, en la zona en la que el arrecife se encuentra más cerca de la costa. La plaza principal, en el centro y cerca de la playa, es un espacio público que reúne tanto a turistas, residenciales o visitantes de un día, como a una parte de la comunidad.

La amplia zona de manglar, cercana a la playa y que continúa hasta la carretera Cancún-Tulum, ha sido una limitante para la expansión de la zona turísticoresidencial de Puerto Morelos, que se concentra en una estrecha franja del litoral y alberga casas, edificios de departamentos y hoteles, por lo que el mayor crecimiento urbano se ha producido en el lado oeste de la carretera, en la colonia Zetina Gasca, donde vive gran parte de la población local. Esta concentración poblacional diferenciada ha producido una fragmentación social importante en la localidad que se manifiesta en la discriminación y exclusión de grupos locales y en otros problemas como alcoholismo, drogadicción, abandono familiar, embarazos en la adolescencia y deserción escolar (Cruz et al., 2013b).

En la línea costera, las viviendas unifamiliares han ido convirtiéndose en edificios de departamentos, hasta ahora con un límite de tres a cuatro niveles, lo que ha implicado que el paisaje urbano esté libre de grandes conjuntos inmobiliario-turísticos, como en otros destinos, debido a que el plan de desarrollo urbano no ha sido modificado para permitirlo. En los últimos años algunos extranjeros han empezado a sumarse como inmigrantes a la comunidad de Puerto Morelos que vive en la colonia Zetina Gasca, donde el precio de los bienes raíces es más accesible y donde, además, los lotes son más grandes.

\section{Turismo residencial en Puerto Morelos}

El fenómeno que se designa actualmente como turismo residencial (Aledo, 2008; Hiernaux, 2010) está presente en México y el mundo después de la Segunda Guerra Mundial. Posteriormente, experimentó un incremento por la globalización en sus facetas económica y cultural, además de que el fenómeno se ha complejizado al mezclarse con otras formas de movilidad y al empalmarse con actividades meramente inmobiliarias (Huete et al., 2008). En el Caribe mexicano, como en otras regiones del país, el fenómeno ha presentado un importante incremento durante las últimas décadas (Jiménez y Sosa, 2010 y 2011), en especial en lo relacionado con las inversiones turístico-inmobiliarias.

El Instituto Nacional de Estadistica, Geografía e Informática (INEGI) tiene establecido como parámetro de análisis: "vivienda de uso o renta temporal", categoría en la que se incluyen, entre otras, las viviendas destinadas a turismo residencial. En el censo de 2010, en Puerto Morelos se contabilizaron 634 viviendas en esta categoría. El Grupo de Estudios de Segundas Residencias realizó un levantamiento de datos en 2004 que arrojó un resultado aproximado de 142 "unidades condominales", que representaban 285 cuartos dedicados a turismo residencial en Puerto Morelos (citado en Jiménez y Sosa, 2010: 68). La comparación de las cifras de 2004 y 2010 muestra un fuerte crecimiento, aun cuando las metodologías aplicadas en cada caso fueron diferentes y se requiera de algunos ajustes para reforzar su comparabilidad. La oferta de cuartos hoteleros era de 1640 en el año 2004, mientras que sumaban 1931 en 2008, y en 2015 se registraron 5072 (SEDETUR, 2015). A partir de estos datos puede afirmarse que tanto el crecimiento de oferta hotelera, como de vivienda para turismo residencial, se haincrementado significativamente en Puerto Morelos: el $300 \%$ en el caso de los cuartos hoteleros y 4.5 veces en el caso de las viviendas para turistas residenciales. 
La información de inversiones y operaciones inmobiliarias muestra que el crecimiento de Puerto Morelos fue moderado hasta iniciado este siglo, cuando cobró mayor impulso, lo que se expresó en el incremento constante de los precios de los bienes raíces, cuanto este aumento se detuvo, situación similar a la ocurrida en otros destinos del norte del Caribe mexicano como Cancún.

Para el año 2010 Puerto Morelos tenía una población de 9365 habitantes, el 63\% de los cuales nació fuera de Quintana Roo, mientras que el 23\% de los mayores de cinco años vivía en otra entidad en el momento del censo; es decir, presenta una fuerte inmigración (INEGI, 2010). De las 4600 viviendas registradas, el 55\% cuenta con luz eléctrica y el 54\% con agua entubada, lo que muestra un rezago de servicios que se concentra en la colonia Zetina Gasca, la cual está separada del área original del puerto por la carretera, y se expande al oeste de la carretera Cancún-Tulum.

\section{El estudio}

El estudio que se presenta, de carácter exploratorio, derivó de los trabajos de la Red de Investigación Turística en México, cuyas líneas de investigación se enfocan en las dimensiones sociales del turismo. Considerando que el propósito esencial de la investigación cualitativa es explorary describir el entendimientoylasinterpretaciones de los fenómenos sociales por parte de los actores sociales en la forma en que los viven (Ritchie, 2003), el estudio adoptó una aproximación cualitativa, la cual ha sido validada en otros estudios y contextos para el análisis de los impactos del turismo residencial (Mazón, Huete y Mantecón, 2010). Lainvestigación cualitativa no privilegia el número de datos, como la cuantitativa, sino la selección de informantes estratégicos y la profundidad de las respuestas, así como la contextualización de las mismas, lo que aporta valiosos insumos para el análisis, además de que estas técnicas se consideran más horizontales por otorgar una mayor presencia a quienes aportan información. Los resultados obtenidos son procesados y categorizados para poder integrarlos en la conceptualización. Siendo éste un análisis de percepción, se consideró la conveniencia de aplicar la investigación cualitativa.
La técnica principal de investigación fue la entrevista en profundidad, y para la selección de informantes se optó por un muestreo no probabilístico. Ciertamente, este tipo de muestreo no pretende realizar inferencias en torno a la población total de estudio, sino que el análisis y las conclusiones, de acuerdo con el enfoque cualitativo, derivan de la información con las características señaladas en el párrafo anterior, y de la organización, categorización y conceptualización que se realicen. El muestreo no probabilístico es de gran utilidad porque ofrece una idea de la variedad de respuestas que los miembros de una población puedan tener y porque ayuda a conseguir un entendimiento profundo del tema a estudiar por la información detallada en las propias palabras y experiencias de los informantes (Altinay y Paraskevas, 2008). Se realizaron 31 entrevistas a residentes permanentes de Puerto Morelos en octubre y noviembre de 2014. Con la intención de considerar varias perspectivas, se puso especial atención en incorporar perfiles sociodemográficos distintos. Participaron 17 mujeres y 14 hombres, con una edad promedio de 43 años y con una media de 17 años de residencia en el destino. En cuanto a su lugar de origen, la mayoría procedía de otras entidades de la república mexicana, algunos del estado de Quintana Roo y tres del extranjero. De igual forma, tenían ocupaciones distintas, con y sin dependencia laboral directa del turismo, condición que permitiría, desde la teoría del intercambio social, comprender si la aceptación de los cambios generados por este tipo de turismo y el apoyo a la actividad dependían del tipo de beneficios recibidos. Participaron en el estudio prestadores de servicios turísticos, artesanos, comerciantes, gerentes, profesores, servidores públicos y amas de casa, por mencionar algunas profesiones, que tenían niveles distintos de escolaridad: desde quienes no tenían ningún nivel de educación formal, hasta otros que contaban con estudios de posgrado - maestría - También se procuró incorporar a informantes residentes tanto de "el lado del Puerto", como de la colonia Zetina Gasca.

Como instrumento de investigación se utilizó una guía de entrevista con preguntas abiertas con las que se perseguía conocer cómo se describía a los turistas residenciales: quiénes eran, su procedencia, su tiempo 
de estancia y el tipo de propiedad que poseían, así como los beneficios y costos — sociales, económicos y en el entorno natural- que su presencia producía tanto a nivel personal, como comunitario. Todas las entrevistas fueron audiograbadas previo consentimiento de los informantes. Se diseñó también un segundo instrumento cuyo objetivo fue recolectar datos generales tanto de la entrevista —número de registro, lugar y fecha donde se realizó-, como de los informantes — lugar de origen, tiempo de residencia, edad, género, ocupación, escolaridad, estado civil y número de hijos-. De 2014 a 2016 se realizaron también visitas y recorridos por las distintas zonas de la comunidad con la intención de recabar datos a través de la observación.

El análisis de las entrevistas se fundamentó en categorías correspondientes a losimpactos socioculturales del turismo. El discurso de cada entrevista fue analizado detenidamente identificando conceptos, descripciones y casos ilustrativos que coincidieran con los indicadores de impactos reportados por la literatura revisada - entre ellos, composición demográfica, multiculturalidad y dinámica inmobiliaria-. A su vez, se reconocía que los impactos del turismo dependen también de las condiciones socioeconómicas y culturales de la población local (Wall y Mathieson, 2006), y que las entrevistas cualitativas dan voz a las subjetividades y vivencias particulares de los informantes, por lo que se buscó identificar otros indicadores que, aunque no ubicados en la bibliografía, fueran reportados por los entrevistados como cambios generados por el turismo residencial en su contexto específico. Se efectuó entonces un análisis guiado por categorías derivadas de la bibliografía, pero a la vez con apertura a las percepciones particulares de los informantes. Los hallazgos fueron interpretados desde las postulaciones de la teoría del intercambio social.

\section{Hallazgos}

Quiénes son los turistas residenciales

El estudio reveló que existen dos tipos de turistas asociados a las segundas residencias; por un lado, extranjeros con propiedad en la localidad y residencia permanente y, por otro, extranjeros con propiedad en la localidad, pero con residencia temporal; en estos últimos se centró el estudio, por lo que se puso especial atención en que los informantes se refirieran a ellos y no a los extranjeros con residencia permanente.

La población local coincidió ampliamente en quiénes eran los turistas residenciales; principalmente adultos mayores jubilados que viajan ya sea en pareja o en familia, provenientes en su mayoría de Canadá, aunque más recientemente también han llegado de Estados Unidos y Europa. En Puerto Morelos este fenómeno es marcadamente estacional, siendo durante la temporada invernal cuando se concentra la mayor actividad y predominan los visitantes extranjeros, que suelen permanecer de uno a cinco meses, entre noviembre y marzo, principalmente, lo que se explica, de acuerdo con los informantes, por las bajas temperaturas en los países del norte.

Estos turistas poseen casas, condominios, departamentos o residencias en Puerto Morelos, y hacen básicamente dos usos de estas propiedades. Pueden acondicionarlas para ofrecer servicios de hospedaje, de manera que rentan los inmuebles a otros visitantes extranjeros, comúnmente del mismo país, durante los periodos en que ellos no los ocupan, o pueden utilizarlos únicamente para su estancia, por lo que son habitados únicamente en temporada invernal; para estos turistas, su residencia en el destino es, en palabras de algunos informantes, "su segundo hogar".

\section{Impactos del turismo residencial a nivel personal}

Uno de los intereses del estudio fue conocer los impactos del turismo residencial percibidos a nivel personal porque, si bien la teoría del intercambio social se ha utilizado preferiblemente en el análisis del intercambio colectivo, la percepción a nivel personal puede definir en gran medida la postura de los pobladores ante la actividad turística. En este sentido, la importancia económica del fenómeno fue reportada como un beneficio específicamente por once informantes debido a su relación directa o indirecta con negocios del sector. Las personas que regentaban 
negocios de alimentos y bebidas, como pequeños restaurantes y cafés, experimentaron un beneficio directo debido a la necesidad de los turistas de adquirir alimentos —no así de hospedaje, lógicamente- para su estancia. Además, otras personas no relacionadas directamente con esta actividad reconocieron que el beneficio económico que recibían algunos prestadores directamente del turista residencial se reflejaba en otros miembros de la comunidad. Esto sugeriría que no sólo el beneficio económico que obtienen los pobladores locales, al menos claramente percibido, es una condición para que apoyen al turismo residencial, porque también reconocen ser beneficiados a través del efecto multiplicador que produce, el cual consiste en que el impacto de cualquier gasto turístico en la economía local se extiende más allá de los receptores iniciales, en este caso, a algunos prestadores de servicios (Duffield y Long, 1981: 412).

Demaneradirectanosoybeneficiadoeconómicamente porque no recibo un sueldo de ellos, pero de manera indirecta claro que sí, porque es lo que mueve a la colonia al final de cuentas. La gente local es la que compra aquí en el negocio, y el turismo que viene ocasionalmente reactiva ese motor económico (Armando).

En cuanto a las desventajas del fenómeno a nivel personal, es muy relevante que, aunque no todos los informantes tenían relación con el turismo residencial y tampoco reconocieron beneficiarse indirectamente, ninguno de los 31 percibía desventajas de cualquier índole. Entonces, si es verdadera la postulación de la teoría del intercambio social de que cuando los costos son menores que los beneficios se produce un apoyo a la actividad, en el caso de estudio se esperaría, por tanto, el apoyo a la actividad turística residencial.

\section{Impactos del turismo residencial a nivel comunitario}

La mayoría de los informantes coincide en que el beneficio económico del fenómeno gira en torno a los inmuebles, la generación de empleo, el consumo en empresas de alimentos y bebidas y, en contadas ocasiones, la compra de servicios turísticos. El empleo se enmarca en la necesidad de cuidar, atender y dar mantenimiento a las propiedades durante el periodo de ausencia de los turistas, quienes "contratan" a personas locales de confianza para que se hagan cargo del cuidado y mantenimiento del inmueble - pagar la luz y el agua, limpieza y jardinería, por ejemplo-. Se produce también un beneficio económico por el consumo que los turistas realizan durante su estancia en el destino por la compra de alimentos y otros productos de consumo diario, no necesariamente en empresas de la industria turística. También algunos involucrados en la prestación de servicios turísticos se benefician porque los turistas residenciales en ocasiones vienen acompañados por otros visitantes que compran servicios, como tours, que generan ingresos para la comunidad, aunque el beneficio se concentra mayormente en los locales que regentan empresas de servicios turísticos.

De esta forma, se presume que el turismo residencial tiene un impacto económico, aunque en su mayoría muy dirigido a segmentos particulares de la población, durante todo el año, a través de la contratación de personal que cuida las casas durante la ausencia de los turistas, y del consumo que realizan durante su presencia. Es importante mencionar que, aunque muchos informantes son conscientes de que sólo algunos se benefician directamente, otros reconocen que se produce un efecto multiplicador de manera que muchas más personas resultan beneficiadas en Puerto Morelos.

Desde las postulaciones de la teoría del intercambio social, los beneficios del turismo residencial pueden comprenderse principalmente a partir de las reglas de la negociación. Es evidente que los beneficios económicos se traducen en las reacciones satisfactorias de los locales. Si se considera que los intercambios negociados son frecuentemente parte de transacciones económicas, en el caso del turismo residencial, y quizá de muchas otras formas de turismo, la satisfacción experimentada por los locales deviene precisamente del valor económico que el fenómeno representa para la vida cotidiana individual y comunitaria de los locales (Cropanzano y Mitchell, 2005). 
Los residentes permanentes parecen estar conscientes también de las desventajas que esta actividad ha traído a sus vidas en el ámbito económico. En este sentido, el estudio reveló que el precio de las propiedades se ha incrementado y suelen cotizarse en dólares, no en pesos mexicanos, lo que sitúa en desventaja a las personas locales para la compra de propiedades porque su poder adquisitivo se torna incompetente ante el poder de la moneda extranjera.

Las propiedades sí subieron [de precio] de una manera exorbitante. Hace treinta años las propiedades de este lado, donde están ahora las residencias, estaban a precios alcanzables para cualquier nacional, pero ahorita son exorbitantes los precios, por eso se da el fenómeno de que los que invierten ahí son más que nada extranjeros cuya moneda es fuerte (Martha, profesora, trece años residente en Puerto Morelos).

Considerando lo que han revelado otros estudios (Duffield y Long, 1981), esta situación puede llevar a una resistencia de las personas locales hacia los foráneos por el hecho de que su presencia crea competencia por las propiedades al causar que los precios se incrementen más allá del alcance de muchos residentes locales. En casos como éste, el intercambio no es percibido como satisfactorio, sino como desigual, lo que se refleja en limitados beneficios económicos para ellos, mientras que resulta favorable para los extranjeros.

En el mismo marco de competencia, el hecho de que los turistas renten sus propiedades a otros visitantes es una desventaja para muchos locales que ofrecen servicios de hospedaje porque el ingreso generado por nuevos visitantes permanece en gran medida en manos de los turistas residenciales. Finalmente, es importante notar que son muy limitados los beneficios económicos que produce el consumo de otros servicios turísticos por parte de los turistas residenciales, en el entendido de que éstos no realizan actividades propiamente turísticas durante su estancia en el destino.

De acuerdo con la teoría del intercambio social, los recursos sobre los que se fundamentan los intercambios, y con ellos las actitudes de residentes hacia turistas y viceversa, no son únicamente de carácter material, sino que también pueden poseer un valor simbólico o inmaterial. En este marco se encuentran los beneficios culturales, que son muy diversos, aunque bien definidos. Una de las ventajas percibidas es la participación de los turistas residenciales en la difusión de actividades culturales en la comunidad, porque estos juegan un papel importante en la organización de eventos musicales, de danza, pintura o teatro, entre otros. Coordinan también charlas sobre temáticas diversas, y otras actividades como cursos de yoga y de inglés para la comunidad en general. Otro beneficio tiene que ver con la multiculturalidad que este tipo de turismo introduce en Puerto Morelos porque, aunque no todos los turistas residenciales se integran a la comunidad - particularmente algunos estadounidenses que crean sus propios grupos-, por lo general favorecen el intercambio cultural en su convivencia con los residentes permanentes, lo que expone a los grupos interactuantes a nuevas formas de pensamiento y, en palabras del informante Uriel, quien ha residido toda su vida en el destino, "al contacto con otros mundos". Las valoraciones positivas de los impactos culturales del turismo residencial conforman en este caso, a diferencia de los impactos económicos, el valor simbólico de las interacciones. Esto demuestra que los intercambios sociales pueden generarse a partir de los recursos simbólicos, que representan un beneficio intangible pero apreciado, más allá del valor objetivo de los mismos.

De la misma manera, los apoyos de alguna forma altruistas a miembros de la comunidad o a espacios comunitarios se observan como beneficios. El estudio reveló que este tipo de turistas obsequian artículos como zapatos y ropa a la gente necesitada, y dulces y juguetes a los niños; también participan activamente en la limpieza, cuidado, mantenimiento y acondicionamiento de espacios públicos como la biblioteca, la casa de cultura, las escuelas, playas y jardines. Finalmente, los turistas residenciales aportan a la dinamización del pueblo en temporada baja.

A pesar de lo anterior, los residentes locales, aunque en menor medida, también son conscientes de los 
costos sociales que conlleva la presencia de este tipo de visitantes, algunos de los cuales son: el desplazamiento de los residentes originarios, especialmente de aquellos que han vendido sus propiedades a los extranjeros, hacia otros espacios alejados del puerto; la privatización de espacios, entre ellos de algunas playas, que son un lugar importante para la recreación de los habitantes locales; la presencia creciente de extranjeros en Puerto Morelos, junto con otros factores como los procesos de urbanización, han exigido el crecimiento de negocios mayores (supermercados) que, si bien pueden significar un beneficio para algunos por la ampliación de oportunidades para la compra de productos básicos, ha representado una desventaja y una amenaza para los pequeños comerciantes y los negocios locales. De acuerdo con la percepción de los informantes, los pequeños negocios tradicionales, las "tienditas", no son competentes ante la presencia de estas grandes tiendas, lo que ha llevado a la desaparición de algunas de ellas.

Por otro lado, las tradiciones se exponen a procesos de transformación como consecuencia de las costumbres principalmente de los norteamericanos - por ejemplo, el festejo de Halloween es cada vez más frecuente en la comunidad - La multiculturalidad a su vez es vista como una desventaja por algunos informantes, quienes señalan que la presencia de personas de distintas culturas ha diluido la imagen del pueblo pesquero tradicional de Puerto Morelos, en donde la identidad particular cada vez se observa menos. Existe, asimismo, una preocupación por parte de los residentes porque el número de extranjeros continúa creciendo, de modo que podría llegar a ser mayor al número de locales nacionales y, como sugiere Edgar, investigador de 65 años de edad, Puerto Morelos podría llegar a convertirse en "un enclave de extranjeros".

Finalmente, se encontró que los impactos en el entorno natural, tanto en beneficios como en costos, fueron considerados mínimos en la percepción de los entrevistados, de los cuales el 30\% percibió algún beneficio ambiental, y el $87 \%$ no percibió ningún costo asociado. El beneficio principal reportado fue un mayor cuidado del entorno natural por parte de los turistas residenciales, cuidado que puede extenderse en la comunidad porque, a través de charlas y acciones concretas, los turistas buscan incrementar la conciencia local acerca de temas como la recolección y separación de basura y el reciclaje. Otras acciones, como recoger los desechos de los perros en espacios públicos, constituyen también un impacto positivo percibido por los locales.

Los costos ambientales también fueron mencionados, aunque sólo cuatro informantes reportaron algún tipo de desventaja en este sentido. Principalmente se mencionó la afectación a los manglares por la expansión de la industria inmobiliaria. Sin embargo, esto no significa que no existan otros costos ambientales del turismo residencial en Puerto Morelos, sino que no es una preocupación relevante para los residentes permanentes.

\section{Conclusiones}

La percepción de los impactos del turismo residencial en Puerto Morelos, así como la valoración de éstos, presenta en general un balance positivo bajo la perspectiva del intercambio social, que supone la consideración de costos versus beneficios (Jurowski y Gursoy, 2004). Particularmente, este balance positivo se explica no sólo a partir de los beneficios materiales económicos del fenómeno, sino también de los beneficios simbólicos culturales que se generan en los procesos de interacción inmaterial entre locales y turistas. Los hallazgos refieren principalmente repercusiones en lo individual, pero también incorporan valoraciones sobre lo colectivo. Al mismo tiempo, se observa claridad y consistencia en relación con los ámbitos económico, social y ambiental, por lo que es posible considerar que las posiciones frente al fenómeno se encuentran bastante definidas. La selección de informantes que cubrían diversos perfiles, las entrevistas a profundidad que proporcionaron información vasta, y la contextualización de la misma, permitieron el análisis confiable de la información que condujo a estas conclusiones.

El ámbito más amplia y claramente tratado por los entrevistados fue el económico. Los impactos económicos del turismo residencial en su mayoría son positivos y se explican por el impulso económico 
del turismo residencial y por el gasto que realizan los turistas. Los impactos negativos - precios de bienes raíces y demanda de bienes de consumo, que propician la creación de negocios no locales - también fueron reconocidos, pero con menor importancia. La percepción de los impactos positivos y negativos es considerada por la teoría del intercambio como valoración de la suma costo/beneficio (Jurowski y Gursoy, 2004; Haley, Snaith y Miller, 2005). Llama la atención que, si bien las repercusiones económicas negativas son definidas con certeza, éstas se refieren a impactos en lo colectivo, no en lo individual, lo que muestra un sentido de comunidad.

Los impactos sociales percibidos permiten también confirmar la claridad con la que se observan actitudes negativas - convivencia exclusiva con otros turistas residenciales extranjeros, por ejemplo-, al igual que otras generadoras de impactos positivos - compromiso para apoyar actividades culturales o acciones comunitariasEl balance final positivo no impide distinguir aquellas que pueden tener repercusiones negativas y positivas a la vez, como la multiculturalidad, que implica apertura y tolerancia pero al mismo tiempo procesos de aculturación o efecto demostración que pueden desdibujar la identidad local.

Resulta notoria la poca atención brindada a los impactos ambientales, a pesar del activismo en este tema que caracteriza a la comunidad de Puerto Morelos. No obstante, la explicación podría encontrarse en que, desde su perspectiva, los entrevistados identifican cómo los turistas residenciales comparten con los locales pronunciamientos y actitudes comunes, y no los relacionan con las problemáticas que tradicionalmente han sido parte de sus preocupaciones ambientales. Con ello, el balance también es positivo en este tema.

A partir de estos resultados, puede entenderse que, en el análisis general sobre los impactos del turismo residencial en Puerto Morelos, los entrevistados encuentran como bueno el balance o resultado total. Una cuarta parte de los informantes reconoce tanto los beneficios, como las desventajas del fenómeno; es decir, no lo descalifican sino que exponen cómo conviven impactos positivos y negativos. Esta información puede ser utilizada para la gestión pública en el desarrollo de estrategias para conciliar el desarrollo comunitario y el turismo.

Desde la perspectiva de la teoría del intercambio social, se confirma que la comunidad valora los costos y los beneficios, en lo personal y para la comunidad, para establecer un balance y definir su posición frente al fenómeno. Es en este sentido como en la comunidad de Puerto Morelos, aun reconociendo los impactos negativos, se acepta el fenómeno. El hecho de que predomine una apreciación positiva representa una ventaja para el desarrollo del turismo residencial, siempre y cuando los beneficios, al menos los percibidos, no sean rebasados por las desventajas que el fenómeno pueda traer a los residentes permanentes. Desde una perspectiva sociocultural, la actitud favorable local puede ser aprovechada para fortalecer las relaciones culturales y con ello minimizar las posibilidades de conflicto y maximizar la integración social entre los distintos grupos - turistas y población local - que residen en el destino.

La valoración realizada por los residentes entrevistados en Puerto Morelos puede orientar sobre las políticas públicas que podrían propiciar un mejor balance costo/beneficio; una de ellas podría ser: el apoyo a pequeñas y medianas empresas locales como mecanismo para impulsar y mantener el efecto económico multiplicador, en lugar de favorecer a grandes empresas de capital foráneo, nacional o internacional. Esto puede beneficiar a las pequeñas y medianas empresas, pero también puede ocasionar mayor derrama económica y la creación o mantenimiento de cadenas productivas.

No obstante, considerando que cada modelo de desarrollo potencia impactos económicos y sociales particulares (Demajorovic et al., 2011), es relevante el modelo turístico por el que se opte. Puerto Morelos ha sido atractivo para residentes provenientes del extranjero por ser un pequeño poblado tranquilo y acogedor. Su conversión a cabecera municipal en 2016 implica autonomía para planear su desarrollo urbano y turístico. Las decisiones de la nueva administración municipal definirán si la planeación y la gestión turística estarán dirigidas a organizar un destino turístico de baja densidad o darán un giro hacia el crecimiento bajo 
el modelo masivo. El impacto del modelo masivo sobre el mercado y los precios de los bienes inmuebles sería mayor que el expuesto por los entrevistados.

Con relación a los impactos sociales, un mecanismo del que la administración pública puede disponer para propiciar mayor integración de residentes y turistas residenciales es el respeto a los espacios públicos y su ampliación como áreas de encuentro y coincidencia, así como el desarrollo de actividades culturales en ellos, lo que reforzará su efecto integrador. Ya la Organización Mundial del Turismo (OMT, 1997) ha explicado que la interacción en espacios públicos es importante porque el residente se encuentra en posibilidades de diálogo o convivencia amistosa con el turista, posición diferente a la de servicio cuando trabaja en instalaciones turísticas. Por el contrario, es necesario no propiciar áreas de exclusión, reservadas para los turistas (Barton, 2006).

La búsqueda de consensos con la población local es también una estrategia recomendable. Experiencias previas de participación de la población para resolver problemas en Puerto Morelos estarían reforzando esta idea; como ejemplo se puede citar el Parque Nacional Arrecife de Puerto Morelos, quefue decretadoárea natural protegida en 1998 como resultado de una iniciativa y de una "activa participación de la comunidad de Puerto Morelos" (INE, 2000: 13).

Finalmente, es necesario reconocer que los hallazgos de este estudio no se pueden generalizar a la población total. Los hallazgos y conclusiones deben ser aplicables únicamente al número de informantes, así como al tiempo y espacio en que el estudio se realizó. Si bien quedan vacíos pendientes por atender, la adopción de otras técnicas de investigación sobre el mismo tema ofrece una oportunidad de investigación per se. Técnicas cualitativas como la etnografía, por ejemplo, permitirían conocer en detalle los efectos del turismo residencial sobre la vida cotidiana tanto de los turistas, como de los residentes. Asimismo, la adopción de aproximaciones cuantitativas al tema puede ser de gran utilidad para confirmar, rechazar o complementar los hallazgos aquí presentados y ofrecer datos duros para la toma de decisiones sobre los impactos del fenómeno en Puerto Morelos y en otros destinos.

\section{Notas}

${ }^{1}$ Ver por ejemplo: Andriotis y Vaughan (2003), Jurowski y Gursoy (2004), McGehee y Andereck (2004), Andereck et al. (2005), Bujosa y Rosselló (2007) y Nunkoo y Gursoy (2012).

${ }^{2}$ Ver por ejemplo: Hall y Müller (2004), Hiernaux (2005), Huete, Mantecón y Mazón (2008), Demajorovic et al. (2011), Hidalgo y Zunino (2011), Mazón, Huete y Mantecón (2011) y Huete y Mantecón (2012).

\section{Referencias}

Aledo, Antonio (2008). "De la tierra al suelo: la transformación del paisaje y el nuevo turismo residencial". En ARBOR Ciencia, Pensamiento y Cultura, 729, enero-febrero: 99-113.

Aledo, Antonio, Hugo García y Guadalupe Ortiz (2010). "Análisis de mapas causales de impactos del turismo residencial". En EMPIRIA. Revista de Metodología de Ciencias Sociales, 20, julio-diciembre: 61-86.

Altinay, Levent y Alexandros Paraskevas (2008). Planning Research in Hospitality and Tourism. Oxford: Butterworth-Heinemann.

Andereck, Kathleen, Karin M. Valentine, Richard Knopf y Christine A. Vogt (2005). "Residents' Perceptions of Community Tourism Impacts". En Annals of Tourism Research, 32(4): 1056-1076.

Andriotis, Konstantinosy Roger D. Vaughan(2003). "Urban Residents' Attitudes toward Tourism Development: The Case of Crete". En Journal of Travel Research, 42(2): 172-185.

Barton, Jonathan (2006). "Sustentabilidad urbana como planificación estratégica”. En RevistaEure, XXXII(96): 27-45.

Bujosa, Ángel y Jaume Rosselló (2007). "Attitudes toward Tourism and Tourism Congestion". En Région et Développment, 25: 193-207.

CESTUR-SECTUR (2004). Elturismodesegundas residencias en México. México: Centro de Estudios Superiores en Turismo, Secretaría de Turismo Federal.

Cropanzano, R. y Marie S. Mitchell (2005). "Social Exchange Theory: An Interdisciplinary Review”. En Journal of Management, 31(6): 874-900. 
Cruz Coria, Erika, Lilia Zizumbo Villareal, Neptalí Monterroso y Ana Luz Quintanilla (2013a). "La confrontación social por el espacio costero: la configuración de paisajes turísticos en Puerto Morelos, Quintana Roo". En Regióny Sociedad, 56: 127-160.

Cruz Coria, Erika, Lilia Zizumbo Villarreal, Neptalí Monterroso y Ana Luz Quintanilla (2013b). "La transformación de los espacios de vida y la configuración del paisaje turístico en Puerto Morelos, Quintana Roo, México". En Polis, Revista Latinoamericana, 12(34): $307-$ 332.

Deverdum, Marie Eugenie, Maribel Osorio y Alfonso Iracheta (2016). "El turismo residencial en Valle de Bravo, México. Una interpretación de su ciclo de vida". En Investigaciones Turísticas, 11: 30-51.

INE (Instituto Nacional Electoral) (2000). Programa de Manejo Parque Nacional Arrecife de Puerto Morelos. México: INE, Comunidad de Puerto Morelos.

Demajorovic, Jacques, Antonio Aledo, Beatriz Landi y Ana Luisa Mantonavi (2011). "Complejos turísticos residenciales. Análisis del crecimiento del turismo residencial en el Mediterráneo español y en el Litoral Nordestino (Brasil) y su impacto socio-ambiental". En Estudios y Perspectivas en Turismo, 20(4): 772-796.

Duffield, Brian y Jonathan Long (1981). "Tourism in the Highlands and Islands of Scotland: Rewards and Conflicts". En Annals of Tourism Research, 8(3): 403-431. Emerson, Richard M. (1976). "Social Exchange Theory". En Annuacl Review of Sociology, 2: 335-362.

Escobedo, Vanessa Alicia, Maribel Osorio, Irma Cortés y Álvaro López (2015). "El turismo residencial en Malinalco: un destino rural del interior de México". En Teoría y Praxis, 17: 37-70.

Gaviria, Mario (1974a). "La producción neocolonialista del espacio". En Papers. Revista de Sociología, 3: 201-217.

Gaviria, Mario (1974b). España a go-gó: turismo, charter y neocolonialismo del espacio. Madrid: Turner.

Haley, A.J., Tim Snaith y Graham Miller (2005). "The social impacts of tourism: A case study of Bath, UK". En Annals of Tourism Research, 32(3): 647-662.

Hall, C. Michael y Dieter K. Müller (2004). Tourism, Mobility and Second Homes. Between Elite Landscape and Common Ground. Ontario: Channel View Publications.
Hidalgo, Rodrigo y Hugo Marcelo Zunino (2011) "Negocios inmobiliarios en centros turísticos de montaña y nuevos modos de vida. El papel de los migrantes de amenidad existenciales en la Comuna de Pucón, Chile". En Estudios y Perspectivas en Turismo, 20(2): 307-326

Hiernaux, Daniel-Nicolas (2005). "La promoción inmobiliaria y el turismo residencial: el caso mexicano". En Scripta Nova, IX(194).

Hiernaux, Daniel-Nicolas (ed.) (2010). Las segundas residencias en México: un balance. México: Plaza y Valdés.

Huete, Raquel (2010). "Opiniones y actitudes ante el turismo residencial en el sur de la Comunidad Valenciana". En Pasos. Revista de Turismo y Patrimonio Cultural, 8(4): 445-461.

Huete, Raquel y Alejandro Mantecón (2010). “Los límites entre el turismo y la migración residencial. Una tipología". Papers, 95(3): 781-801.

Huete, Raquel y Alejandro Mantecón (2012). "Introducción. Sobre la construcción social de los lugares". En Tomás Mazón, Raquel Huete y Alejandro Mantecón (eds.), Construir una nueva vida. Losespacios del turismo y la migración residencial. Santander: Milrazones, pp. 1l-19.

Huete, Raquel, Alejandro Mantecón y Tomás Mazón (2008). "iDe qué hablamos cuando hablamos de turismo residencial?" En Cuadernos de Turismo, 22, juliodiciembre: 101-121.

INEGI (Instituto Nacional de Estadística y Geografía) (2010). Censo de población y vivienda 2010. México: INEGI

Jiménez, Alfonso y Pricila Sosa (2010). El turismo de segundas residencias en Cancún y la Riviera Maya. México: UAEM, Universidad del Caribe, Plaza y Valdés.

Jiménez, Alfonso y Pricila Sosa (2011). "Del cielo a la tierra y de la tierra al suelo. El turismo residencial en el Caribe Mexicano". En Maciá Blazquez y Ernest Cañada (eds.), Turismo placebo. Nueva colonización turística: del Mediterráneo a Mesoamérica y el Caribe. Nicaragua: Enlace, pp. 275-296.

Jurdao, Francisco (1979). España en venta: compra de suelo por extranjeros y colonización de campesinos en la Costa del Sol. Madrid: Endymion. 
Jurowski, Claudia y Dogan Gursoy (2004). "Distance Effects on Residents' Attitudes toward Tourism”. En Annals of Tourism Research, 31(2): 296-312.

Lizárraga, Omar (2010). "The US Citizens Retirement Migration to Los Cabos, Mexico. Profile and Social Effects". En RASAALA: Recreation and Society in Africa, Asia and Latin America, 1(1): 75-92.

Lizárraga, Omar (2014). "Percepción cultural entre residentes extranjeros y habitantes locales en un destino turístico mexicano. El caso de estadounidenses en Mazatlán, Sinaloa”. En Investigaciones Turísticas, 7: 47-70.

Mantecón, Alejandroy Raquel Huete (2011). “Sociological Insights on Residential Tourism: Host Society Attitudes in a Mature Destination". En European Journal of Tourism Research, 4(2): 109-122.

Mason, Peter (2008). Tourism Impacts, Planning and Management. Londres: Butterhworth-Heinemann.

Mazón, Tomás, Raquel Huete y Alejandro Mantecón (2010). "Tourism Dependence and Host Community Perceptions. Notes on the Social Exchange Theory". En Encontros Científicos - Tourism \& Management Studies, 5: 25-36.

Mazón, Tomás, Raquel Huete y Alejandro Mantecón (2011). Construir una nueva vida. Los espacios del turismo y la migración residencial. Santander: Milrazones.

McGehee, Nancy G. y L. Kathleen Andereck (2004). "Factors Predicting Rural Residents' Support for Tourism". En Journal of Travel Research, 43(2): 131-140.

Negrete, Vicente (2011). "El turismo residencial y la especulación inmobiliaria en Puerto Peñasco”. Tesis de maestría en Arquitectura, México, Universidad Nacional Autónoma de México.

Nunkoo, Robin y Dogan Gursoy (2012). "Resident's Support for Tourism. An Identity Perspective". En Annals of Tourism Research, 33(1): 243-268.

OMT (Organización Mundial del Turismo) (1997). Reunión de líderes del turismo sobre los efectos sociales del turismo. Informe final. Madrid: OMT.
Ramírez, Irma y Maribel Osorio (2010). "Turismo residencial y desarrollo local en el Estado de México". En Daniel-Nicolas Hiernaux (ed.), Las segundas residencias en México: un balance. México: Plaza y Valdés, pp. 145-184.

Ritchie, Jane (2003). "The Applications of Qualitative Methods to Social Research". En Jane Ritchie y Jane Lewis (eds.), Qualitative Research Practice. A Guide for Social Science Students and Researchers. Londres: SAGE, pp. 24-46.

Ryan, Chris (2003). Recreational Tourism: Demands and Impacts. Clevedon: Channel View Publications.

SEDETUR (2015). "Indicadores turístimos enero 2015". Quintana Roo: Secretaría de Turismo del Estado de Quintana Roo. Disponible en: http://sedetur. qroo.gob.mx/estadisticas/indicadores/indicadoresturisticos-2015.pdf (consultado el ll de junio de 2016).

Schafran, Alex y Paavo Monkkonen (2011). "Beyond Chapala and Cancún: Grappling with the Impact of American Migration to Mexico". En Migraciones Internacionales, 6(2): 223-258.

Serrano, José María (2003).“Las viviendas de segunda residencia en la sociedad del 'bienestar'. El caso de un país turístico: España”. En Cuadernos de Turismo, 12: 53-75.

Sharpley, Richard (2015). "Host Perceptions of Tourism: a Review of the Research". En Tourism Management, 42 37-49.

Talavera, Francisco (1982). Lago de Chapala. Turismo residencial y campesinado. Guadalajara: Instituto Nacional de Antropología e Historia.

Wall, Geoffrey y Alister Mathieson (2006). Tourism. Change, impacts and opportunities. Essex: Pearson Prentice Hall.

Ward, Colleen y Tracy Berno (2011). "Beyond Social Exchange Theory. Attitudes towards Tourists". En Annals of Tourism Research, 38(4): 1556-1569. 
Mapa 1. Ubicación de Puerto Morelos, Quintana Roo, México

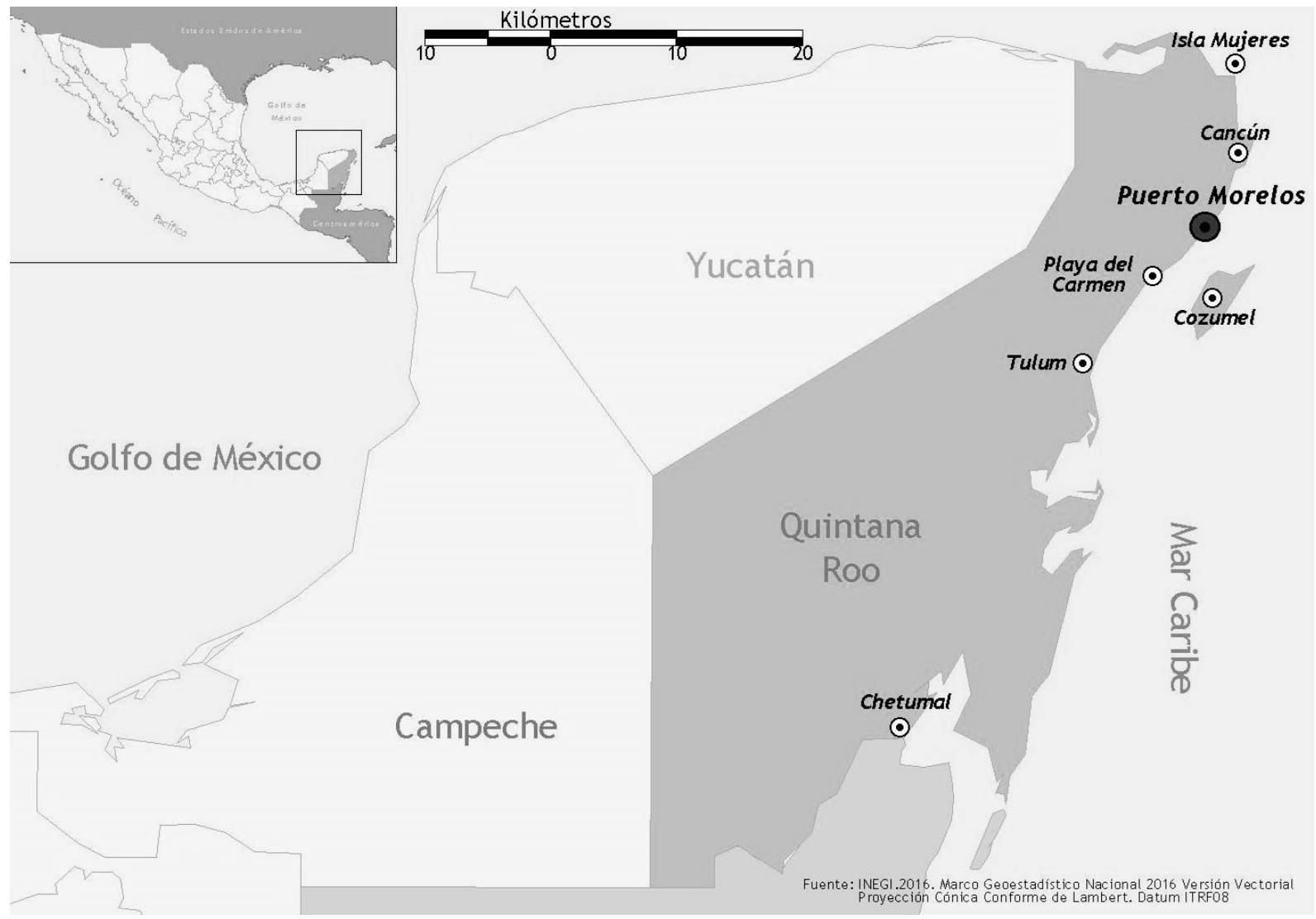

Fuente: elaborado por el Dr. Héctor Hugo Regil García. 